\title{
ADAPTABILIDADE E ESTABILIDADE DE HÍBRIDOS DE MILHO EM DIFERENTES CONDIÇÕES AMBIENTAIS DO NORDESTE BRASILEIRO
}

\author{
HÉLIO WILSON LEMOS DE CARVALHO ${ }^{1}$, MARIA DE LOURDES DA SILVA LEAL ${ }^{1}$, MILTON \\ JOSÉ CARDOSO ${ }^{2}$, MANOEL XAVIER DOS SANTOS ${ }^{3}$, JOSÉ NILDO TABOSA ${ }^{4}$, DENIS \\ MEDEIROS DOS SANTOS ${ }^{1}$, MARCELO ABDON LIRA ${ }^{5}$
}

\author{
${ }^{1}$ Embrapa Tabuleiros Costeiros. Caixa Postal 44, CEP.49001-970Aracaju, SE.E-mail: helio@cpatc.embrapa.br(au- \\ tor para correspondência) \\ ${ }^{2}$ Embrapa Meio-Norte. Caixa Postal 001, CEP.64006-220 Teresina, PI \\ ${ }^{3}$ Embrapa Milho e Sorgo. Caixa Postal 151, CEP.35701-970 Sete Lagoas, MG \\ ${ }^{4} I P A$. Caixa Postal 1022, CEP.50761-000 Recife, PE. \\ ${ }^{5}$ Emparn. Rua Major Laurentino de Moraes, 1220, CEP.59020-390 Natal, RN
}

Revista Brasileira de Milho e Sorgo, v.1, n.2, p.75-82, 2002

\begin{abstract}
RESUMO - Quarenta e um híbridos de milho foram submetidos a vinte e cinco diferentes condições ambientais do Nordeste brasileiro, no ano agrícola de 1999/2000, visando conhecer a adaptabilidade e a estabilidade de produção de grãos desses materiais, para fins de recomendação na região. Utilizou-se o delineamento experimental em blocos ao acaso, com três repetições. A análise de variância conjunta mostrou diferenças marcantes entre os híbridos e a existência de diferenças genéticas entre os híbridos quanto às respostas às variações ambientais. A produtividade média apresentada pelos híbridos $\left(6.971 \mathrm{~kg} \mathrm{ha}^{-1}\right)$ revela que a região mostra condições ambientais favoráveis ao desenvolvimento desses materiais genéticos, especialmente, nas áreas de cerrados do Oeste Baiano, Sul do Maranhão e Pólo UruçuíGurgéia (no Piauí), onde as condições de clima, solo e topografia possibilitam o emprego de tecnologias modernas para a produção de grãos em sequeiro. A maioria dos híbridos mostrou alta estabilidade de produção nos ambientes considerados.
\end{abstract}

Palavras-chave: Zea mays, interação genótipo x ambiente, Nordeste brasileiro, produção de grãos.

\section{ADAPTABILITY AND STABILITY OF MAIZE HYBRIDS IN DIFFERENT ENVIRONMENTALCONDITIONS IN NORTHEASTERN BRAZIL}

\begin{abstract}
During the agricultural year of 1999/2000, forty-one maize hybrids were evaluated in twenty-five different environmental conditions in Northeastern Brazil in order to know their adaptability and the stability of grain yield for planting recommendations. The experimental design was randomized blocks with three replications in each location. The combined analysis of variance showed strong differences among the hybrids within each environment, as well as genetic differences among the hybrids due to environmental variations. The mean grain yield of the hybrids $\left(6.971 \mathrm{~kg} \mathrm{ha}^{-1}\right)$ reveals that the region shows favorable environmental conditions for planting those genetic materials especially in the cerrado areas of Western Bahia, Southern Maranhão and in the Uruçuí - Gurgéia region (Piauí State), where the climate, soil and topography facilitate the use of modern technologies for the production of grains in dry land areas. Most of the hybrids showed high production stability in the considered environments.
\end{abstract}

Key words: Zea mays, genotype x environment interaction, Brazilian Northeast, grain yield 
A partir da década de 1980, iniciou-se a exploração comercial do milho nos cerrados nordestinos, localizados no Oeste Baiano, Sul do Maranhão e no Pólo Uruçuí-Gurgéia, localizado no Estado do Piauí. Essas áreas ocupam um pouco mais de um milhão de hectares e são propícias ao desenvolvimento do milho, por apresentarem condições de solo e clima privilegiados para a produção de grãos em sequeiro, além de exibirem topografia que possibilita a instalação de uma agricultura mecanizada e emprego de alta tecnologia na produção de grãos. Tem-se observado, por outro lado, um incremento considerável na demanda pelo milho no Nordeste brasileiro, em razão da alta densidade demográfica e do crescente aumento da exploração de aves e suínos, tornando necessária, até mesmo, a importação de grande quantidade de milho de outras partes do país e do exterior, para complementar a necessidade regional, em razão da produção do Nordeste brasileiro não ser suficiente para suprir a sua demanda.

Anualmente, diversas instituições públicas e privadas têm desenvolvido e recomendado híbridos de milho que associam boa adaptação a atributos agronômicos desejáveis. Todavia, segundo Ribeiro et al. (1999), um dos grandes problemas que surgem é a inconsistência no comportamento desses materiais, frente às variações ambientais, expressa pela interação genótipos e ambientes. Essa interação assume papel fundamental no processo de recomendação de cultivares, havendo necessidade de atenuar os seus efeitos, através da identificação de cultivares com maior estabilidade fenotípica (Ramalho et al., 1993). A presença significativa dessa interação tem sido constatada em diversas oportunidades, em diferentes regiões do país, conforme relatam Arias (1996), no Estado do Mato Grosso, Carneiro (1998), no Estado do Paraná, Ribeiro et al. (1999), em diferentes condições ambientais no Estado de Minas Gerais, Cardoso et al. (1997 e 2000), na região Meio-Norte do Brasil, Monteiro et al. (1998), no Estado do Ceará e Carvalho et al. (1998c e 2000b), em diversos anos e locais do Nordeste brasileiro. Em todos esses casos, os autores mencionados procuraram minimizar o efeito da interação genótipos e ambientes, recomendando materiais de melhor estabilidade fenotípica.

Considerando esses aspectos, este trabalho visou conhecer a adaptabilidade e a estabilidade de quarenta e um híbridos de milho, quando submetidos a diferentes condições ambientais no Nordeste brasileiro, para fins de recomendação.

\section{Material e Métodos}

Os ensaios, em regime de sequeiro, foram realizados em 23 ambientes do Nordeste brasileiro, distribuídos nos Estados do Maranhão (quatro ensaios), Piauí (sete ensaios), Rio Grande do Norte (um ensaio) Pernambuco (quatro ensaios), Alagoas (um ensaio), Sergipe (um ensaio) e Bahia (cinco ensaios), com plantios efetuados entre novembro de 1999 e maio de 2000 (Tabela 1). Foram realizados, ainda, dois ensaios sob regime de irrigação, nos municípios de Teresina e Parnaíba, no Piauí, com plantio efetuado em junho/2000.

Na Tabela 1, estão os índices pluviais (mm) registrados no decorrer do período experimental, com uma variação de $310,8 \mathrm{~mm}$ a $1.643,6 \mathrm{~mm}$. Na Tabela 2, constam as coordenadas geográficas de cada município, os quais estão compreendidos entre os paralelos $2^{\circ} 53^{\prime}(\mathrm{S})$ e $14^{\circ} 36^{\prime}(\mathrm{S})$, englobando diferentes condições ambientais (Silva et al., 1993).

Foram utilizados quarenta e um híbridos, sendo dezenove híbridos simples, dezesseis híbridos triplos e seis híbridos duplos. Utilizou-se o delineamento experimental em blocos casualizados, com três repetições. Cada parcela constou de quatro fileiras de 5,0 $\mathrm{m}$ de comprimento, espaçadas de $0,90 \mathrm{~m}$ e $0,50 \mathrm{~m}$ entre covas. Foram colocadas três 
sementes por cova, deixando-se duas plantas por cova após o desbaste. Foram colhidas as duas fileiras centrais de forma integral, correspondendo a uma área útil de $9,0 \mathrm{~m}^{2}$. As adubações realizadas em cada experimento obedeceram aos resultados das análises de solo de cada área experimental.

Os pesos de grãos, após serem ajustados para $15 \%$ de umidade, foram submetidos a análise de variância, obedecendo ao modelo em blocos casualizados. Após a análise de cada ensaio, efetuou-se a análise de variância conjunta, obedecendo ao critério de homogeneidade dos quadrados médios residuais. As referidas análises foram efetuadas utilizando-se o Statistical Analysis System (SAS Institute, 1996) para dados balanceados (PROC ANOVA).

TABELA 1. Índices pluviais (mm) ocorridos durante o período experimental. Região Nordeste do Brasil; 1999/2000.

\begin{tabular}{|c|c|c|c|c|c|c|c|c|c|c|c|}
\hline \multirow{2}{*}{ Locais } & \multicolumn{2}{|c|}{1999} & \multicolumn{8}{|c|}{2000} & \multirow{2}{*}{ Totais } \\
\hline & Nov. & Dez. & Jan. & Fev. & Mar. & Abr. & Mai. & Jun. & Jul. & Ago. & \\
\hline S. R. Mangabeira & - & $351,3^{*}$ & 366,4 & 401,6 & 364,5 & 159,8 & - & - & - & - & 1643,6 \\
\hline Sambaiba & - & $347,0^{4}$ & 231,0 & 399,0 & 245,0 & 93,0 & - & - & - & - & 1315,0 \\
\hline Barra do Corda & - & $235,8^{*}$ & 139,0 & 212,6 & 266,0 & 214,4 & - & - & - & - & 1067,8 \\
\hline Anapurus & - & - & $207,0^{*}$ & 254,0 & 321,0 & 426,0 & 245,0 & - & - & - & 1453,0 \\
\hline Teresina & - & - & $306,8^{*}$ & 329,8 & 298,4 & 68,4 & 6,7 & - & - & - & 1010,1 \\
\hline Parnaiba & - & - & $166,5^{4}$ & 233,9 & 157,5 & 391,5 & 201,1 & - & - & - & 1150,5 \\
\hline Guadalupe & - & - & $173,0^{*}$ & 312,0 & 369,5 & 147,0 & 64,6 & - & - & - & 1066,1 \\
\hline Rio Grande Piaui & - & - & $185,0^{*}$ & 310,0 & 390,4 & 128,2 & 50,1 & - & - & - & 1063,7 \\
\hline Palmeiras Piaui & $345,0^{*}$ & 236,5 & 224,0 & 265,0 & 135,5 & - & - & - & - & - & 1206,0 \\
\hline Bom Jesus & - & 365,0 & 149,5 & 172,0 & 179,0 & - & - & - & - & - & 1131,5 \\
\hline Baixa G. Ribciro & - & $277,5^{*}$ & 173,0 & 364,5 & 367,0 & 91,5 & - & - & - & - & 1273,5 \\
\hline Canguaretama & - & - & - & $147,2^{*}$ & 82,6 & 199,0 & 200,8 & - & - & - & 629,6 \\
\hline Caruaru & - & - & - & - & - & 108,5 & 92,6 & 315,3 & 244,1 & 376,5 & 1136,5 \\
\hline S. Bento do Una & - & - & - & - & - & - & $4,0^{*}$ & 139,2 & 70,6 & 97,0 & 310,8 \\
\hline Serra Talhada & - & - & - & $16,6^{*}$ & 92,9 & 174,9 & 16,4 & 33,2 & - & - & 334,0 \\
\hline Araripina & $\cdot$ & - & $27,6^{*}$ & 133,3 & 106,2 & 106,2 & 2,6 & - & - & - & 375,9 \\
\hline Coruripe & - & - & - & $\cdot$ & $\cdot$ & - & 116 & 255 & 240 & 113 & 724 \\
\hline N. Sra. das Dores & - & - & - & - & - & - & 122,0 & 269,0 & 120,0 & 142,0 & 653,0 \\
\hline Lapão & - & $212,9^{*}$ & 129,4 & 86,9 & 25,0 & - & - & - & - & - & 454,2 \\
\hline Ibititá & - & $207,0^{*}$ & 57,8 & 74,7 & 64,2 & - & - & - & - & - & 403,7 \\
\hline Barra do Choça & $\cdot$ & $38,1^{*}$ & 69,5 & 83,1 & 48,6 & $=$ & - & $=$ & $=$ & - & 239,3 \\
\hline Barreiras 1 & - & $200,0^{4}$ & 163,0 & 285,0 & 219,0 & - & - & - & - & - & 867,0 \\
\hline Barreiras 2 & - & $200,0^{*}$ & 170,0 & 290,0 & 180,0 & - & - & - & - & - & 840,0 \\
\hline
\end{tabular}

*Mês de plantio não registrado. 
TABELA 2. Coordenadas geográficas dos locais e tipos de solo das áreas experimentais.

\begin{tabular}{|c|c|c|c|c|c|}
\hline Estado & Municipio & Latitude (S) & Longitude (W) & Altitude & Tipo de solo ${ }^{1}$ \\
\hline \multirow[t]{4}{*}{ Maranhão } & S. R. Mangabcira & $7^{\circ} 22$ & $45^{\circ} 36$ & - & PVA \\
\hline & Sambaiba & $7^{\circ} 08$ & $45^{\circ} 20^{\prime}$ & - & PA \\
\hline & Barra do Corda & $5^{\circ} 43^{\prime}$ & $45^{\circ} 18^{\circ}$ & - & LA \\
\hline & Anapurus & $3^{\circ} 55$ & $43^{\circ} 30^{\prime}$ & - & LA \\
\hline \multirow[t]{7}{*}{ Piaui } & Teresina & $5^{\circ} 5^{\prime}$ & $42^{\circ} 49^{\prime}$ & 72 & A \\
\hline & Parnaiba & $2^{\circ} 53^{\prime}$ & $41^{\circ} 41^{\circ}$ & 15 & $\mathrm{AQ}$ \\
\hline & Guadalupe & $6^{\circ} 56$ & $43^{\circ} 50^{\circ}$ & 180 & LVA \\
\hline & Rio G. do Piaui & $7^{\circ} 36$ & $43^{\circ} 13^{v}$ & 270 & PVA \\
\hline & Palmeiras Piauí & $8^{\circ} 43^{\prime}$ & $44^{\circ} 14^{\prime}$ & - & LA \\
\hline & Bom Jesus & $9^{\circ} 04$ & $44^{\circ} 21^{\prime}$ & - & LA \\
\hline & Baixa G. Ribeiro & $T^{\circ} 32$ & $45^{\circ} 14^{\circ}$ & - & $\mathrm{AQ}$ \\
\hline Rio G. do Norte & Canguaretama & $6^{\circ} 22$ & $35^{\circ} 07$ & 5 & LVA \\
\hline \multirow[t]{4}{*}{ Pernambuco } & São Bento do Una & $8^{\circ} 31^{\prime}$ & $36^{\circ} 22^{\prime}$ & 645 & $\mathrm{R}$ \\
\hline & Serra Talhada & $8^{\circ} 17$ & $38^{\circ} 29^{\prime}$ & 365 & PVA \\
\hline & Araripina & $7^{\circ} 33$ & $40^{\prime} 34^{\prime}$ & 620 & LVA \\
\hline & Caruaru & $8^{\circ} 34$ & $38^{\circ} 00^{\prime}$ & 537 & PVA \\
\hline Alagoas & Coruripe & & & & \\
\hline Sergipe & N. Sra. Das Dores & $10^{\circ} 30^{\circ}$ & $37^{\circ} 13^{\prime}$ & 200 & LVA \\
\hline \multirow[t]{5}{*}{ Bahia } & Lapão & $11^{\circ} 21^{\circ}$ & $41^{\circ} 41^{\circ}$ & 785 & A \\
\hline & Ibititá & $11^{\circ} 32^{\prime}$ & $41^{\circ} 41^{\prime}$ & 700 & $\mathrm{~A}$ \\
\hline & Barreiras 1 & $10^{\circ} 48^{\prime}$ & $44^{\circ} 41^{\prime}$ & 800 & $\mathrm{AQ}$ \\
\hline & Barreiras 2 & $12^{\circ} 21^{\prime}$ & $44^{\circ} 41^{\prime}$ & 780 & $\mathrm{AQ}$ \\
\hline & Barra do Choça & $14^{0} 36$ & $40^{\circ} 36$ & 880 & PVA \\
\hline
\end{tabular}

${ }^{1}$ A- Aluvial; AQ- Areia Quartzosa; LVA- Latossolo Vermelho-Amarelo; PVA-Podzólico Vermelho-Amarelo; R- Regossolo; LA - Latossolo Amarelo; PA- Podzólico Amarelo.

Os parâmetros de adaptabilidade e estabi- favoráveis $\left(b_{1}+b_{2}\right)$. A estabilidade dos materiais é lidade foram estimados utilizando-se o método de avaliada pelos desvios da regressão $\sigma^{2}$ de cada Cruz et al. (1989), o qual baseia-se na análise de material, de acordo com as variações ambientais. regressão bissegmentada, tendo como parâmetros É utilizado o seguinte modelo: de adaptabilidade a média $\left(\mathrm{b}_{0}\right)$ e a resposta linear $\quad \mathrm{Y}_{\mathrm{ij}}=\mathrm{b}_{\mathrm{oi}}+\mathrm{b}_{1 \mathrm{i}} \mathrm{I}_{\mathrm{j}}+\mathrm{b}_{2 \mathrm{i}} \mathrm{T}(\mathrm{I})+\delta_{\mathrm{ij}}+\mathrm{e}_{\mathrm{ij}}$, aos ambientes desfavoráveis $\left(b_{1}\right)$ e aos ambientes em que: $Y_{\mathrm{ij}}$ : média da cultivar i no ambiente $\mathrm{j}$; $\mathrm{I}_{\mathrm{j}}$ : 
índice ambiental, conforme propuseram Eberhart \& $\operatorname{Russel}(1966) ; \mathrm{T}\left(\mathrm{I}_{\mathrm{j}}\right)=0 \mathrm{se} \mathrm{I}_{\mathrm{j}}<0 ; \mathrm{T}\left(\mathrm{I}_{\mathrm{j}}\right)=\mathrm{i}_{\mathrm{j}} \mathrm{I}_{+} \mathrm{SE}_{\mathrm{j}}>0$, sendo $I_{+}$a média dos índices $I_{j}$ positivos; $b_{0 \mathrm{i}}$ : média geral da cultivar $i ; b_{1 i}$ : coeficiente de regressão linear associado a ambientes desfavoráveis; $\mathrm{b}_{1 \mathrm{i}}+\mathrm{b}_{2 \mathrm{i}}$; coeficiente de regressão linear associado a ambientes

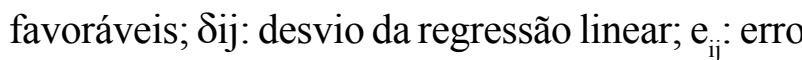
médio associado a média.

\section{Resultados e Discussão}

As análises de variância por ambientes mostraram efeitos significativos entre os híbridos, evidenciando variações genéticas entre eles, em todos os ambientes. Os coeficientes de variação obtidos oscilaram de 6,7\% a 16,4\%, em conseqüência da boa precisão dos ensaios (Scapim et al., 1995). A média de produtividade nos ensaios oscilou de 3.669 $\mathrm{kg} \mathrm{ha}^{-1}$, em Coruripe, a $10.498 \mathrm{~kg} \mathrm{ha}^{-1}$, em Baixa Grande do Ribeiro, o que expressa ampla faixa de variação onde foram realizados os experimentos. Os municípios de São Raimundo Mangabeira, no Maranhão, Teresina, Parnaíba, Rio Grande do Piauí, Palmeiras do Piauí e Baixa Grande do Ribeiro, no Piauí, e Barreiras (local 2), na Bahia, com produtividades médias entre $8.078 \mathrm{~kg} \mathrm{ha}^{-1} \mathrm{e} 10.498 \mathrm{~kg} \mathrm{ha}^{-1}$, destacaram-se como os mais favoráveis para a exploração do milho, ressaltando-se que esses resultados colocam essas áreas em condições de competir com as áreas tradicionais de cultivo de milho do país. Os municípios de Anapurus, no Maranhão, Bom Jesus, no Piauí, e Lapão, na Bahia, também se destacaram, com produtividades médias de grãos acima da média geral. Esses resultados superaram aqueles obtidos em anos anteriores, em diversas localidades do Nordeste brasileiro (Cardoso et al., 1997 e 2000) e Carvalho et al. (2000a e 2000b).

A análise de variância conjunta mostrou efeitos significativos para ambientes, híbridos e interação híbridos ambientes, o que evidencia diferenças entre os híbridos e inconsistência no comportamento dos híbridos em face das oscilações ambientais, justificando-se o estudo da adaptabilidade e estabilidade desses materiais, no detalhamento da interação híbridos e ambientes.

Observou-se que a produtividade média $\left(b_{0}\right)$ variou de $3.749 \mathrm{~kg} \mathrm{ha}^{-1}$ (96 HT 91) a $7.716 \mathrm{~kg}$ ha $^{-1}$ (DK 440), com média geral de $6.971 \mathrm{~kg} \mathrm{ha}^{-1}$, evidenciando alto potencial para a produtividade dos materiais avaliados (Tabela 3). Ressalta-se que, aliado ao modelo proposto, consideraram-se como híbridos melhor adaptados aqueles que expressaram maiores produtividades médias de grãos, ou seja, acima da média geral (Mariotti et al., 1976).

A estimativa de $b_{1}$, que avalia o desempenho dos materiais nos ambientes desfavoráveis, evidenciou que, entre os híbridos de melhor adaptação, o DK 350, Pioneer 30 F 45 e Cargill 747 foram menos exigentes nas condições desfavoráveis $\left(b_{1}<1\right)$. Os híbridos Dina 1000, AG 1051, Zeneca 8420 , Zeneca 8550 e Dina 800 E mostraram-se muito exigentes nas condições desfavoráveis, apresentando estimativas de $b_{1}>1$. A estimativa de $b_{1}+b_{2}$, que avalia o desempenho dos materiais nos ambientes favoráveis, mostrou que, dentre os híbridos que apresentaram rendimentos médios superiores em relação à média geral, apenas o Cargill 333 B, Zeneca 8550 e Zeneca 84 E 90 foram responsivos à melhoria ambiental.

Segundo Cruz et al. (1989), a previsibilidade de comportamento pode ser avaliada pela estimativa de $\mathrm{R}^{2}$, salientando que aqueles materiais que apresentarem estimativas de $\mathrm{R}^{2}$ acima de $80 \%$ não devem ter os seus graus de previsibilidade comprometidos. Desta forma, nota-se que todos os materiais, à exceção do HT 9, expressaram boa estabilidade nos ambientes considerados $\left(\mathrm{R}^{2}>80 \%\right)$, independentemente de suas bases genéticas (híbridos simples, duplos ou triplos). 
TABELA 3. Produtividades médias de grãos $(\mathrm{kg}$ $\mathrm{ha}^{-1}$ ) e estimativas de parâmetros de adaptabilidade e estabilidade de 41 híbridos de milho em 25 ambientes do Nordeste brasileiro, no ano agrícola de 1999/2000.

\begin{tabular}{|c|c|c|c|c|c|c|c|}
\hline \multirow{2}{*}{ Hibridos } & \multicolumn{3}{|c|}{ Médins nos ambientes } & \multirow{2}{*}{$b_{1}$} & \multirow{2}{*}{$b_{2}$} & \multirow{2}{*}{$b_{1}+b_{2}$} & \multirow{2}{*}{$\mathbf{R}^{2}$} \\
\hline & Geral & Desfovorivel & Fworived & & & & \\
\hline DK $440^{1}$ & $7716 \mathrm{a}$ & 5827 & 9227 & $1,01 \mathrm{~ns}$ & $0,17 \mathrm{~ms}$ & $1,08 \mathrm{~ns}$ & 90 \\
\hline Dina $1000^{\prime}$ & $7675 a$ & 5834 & 9375 & $1,14^{* *}$ & $-0,25 \mathrm{~ns}$ & $0,89 \mathrm{~ns}$ & 92 \\
\hline$A G 1051^{3}$ & $7617 \mathrm{a}$ & 5599 & 9480 & $1,20^{* *}$ & $-0,07 \mathrm{~ns}$ & $1,12 \mathrm{~ns}$ & 91 \\
\hline Cargell $333 \mathrm{~B}^{\prime}$ & $7615 a$ & 5938 & 9163 & $1,01 \mathrm{~ns}$ & $0,50 * 0$ & $1,51 * 4$ & 90 \\
\hline DKB $350^{2}$ & $7574 a$ & 6114 & 8921 & $0,89^{*}$ & $0,09 \mathrm{~ns}$ & $0,99 \mathrm{~ns}$ & 90 \\
\hline $\operatorname{AGB} 8080^{2}$ & $7648 a$ & 5773 & 9029 & $1,02 \mathrm{~ns}$ & $-0,29^{*}$ & $0,72^{*}$ & 85 \\
\hline Zeneca $8420^{\circ}$ & $7442 \mathrm{a}$ & 5619 & 9125 & $1,11^{*}$ & $-0,04 n s$ & $1,07 \mathrm{~ns}$ & 91 \\
\hline Zeneca $8550^{\prime}$ & $7414 a$ & 5523 & 9160 & $1,09^{*}$ & $0,24 \mathrm{~ms}$ & $1,34^{* *}$ & 90 \\
\hline AG $9090^{\prime}$ & $7373 a$ & 5577 & 9032 & $1,05 \mathrm{~ns}$ & $-0,06 \mathrm{~ns}$ & $0,98 \mathrm{~ns}$ & 93 \\
\hline Pioneer $30 \mathrm{~K} 75^{1}$ & $7363 a$ & 5703 & 8894 & $0,97 \mathrm{~ns}$ & $=0,25 \mathrm{~ns}$ & $0,71^{*}$ & 87 \\
\hline Pioneer 30 F $33^{1}$ & $7351 a$ & 5574 & 8990 & $1,09 \mathrm{~ns}$ & $-0,15 n s$ & $0,93 n s$ & $8 ?$ \\
\hline Pioneer 30 F $45^{1}$ & $7322 \mathrm{~b}$ & 5741 & 8782 & $0,90^{\circ}$ & $0,14 n s$ & $1,05 \mathrm{~ns}$ & 87 \\
\hline Dina $500^{3}$ & $7235 b$ & 5578 & 8721 & 0,96ns & $-0,28^{*}$ & $0,68^{*}$ & 80 \\
\hline$A G 8020^{\prime}$ & 72206 & 5504 & 8801 & $0,98 n s$ & $-0,09 \mathrm{~ns}$ & $0,88 \mathrm{~ns}$ & 93 \\
\hline Zeneca $8392^{\prime}$ & $7213 b$ & 5508 & 8828 & 1,04 ins & $0,18 \mathrm{~ms}$ & $1,23 \mathrm{~ns}$ & 92 \\
\hline Dina $800 \mathrm{E}^{1}$ & $720 \mathrm{lb}$ & 5144 & 9100 & $1,18=$. & $=0,01 \mathrm{~ns}$ & $1,16 \mathrm{~ns}$ & 91 \\
\hline Cargi $747^{3}$ & $7178 b$ & 5937 & 8325 & $0,79 * *$ & $0,17 \mathrm{~ms}$ & $0,96 \mathrm{~ns}$ & 85 \\
\hline Cargi] $909^{1}$ & $7178 b$ & 5596 & 8642 & $0,94 \mathrm{~ns}$ & $0,28^{\circ}$ & $1,23 \mathrm{~ns}$ & 88 \\
\hline Zeneca $8330^{2}$ & $7135 b$ & 5604 & 8549 & $0,91 n$ s & $0,19 \mathrm{~ms}$ & $1.10 \mathrm{~ns}$ & 95 \\
\hline Zeneca 84 E $90^{1}$ & $7071 \mathrm{~b}$ & 5296 & 8700 & $1,05 n s$ & $0,52^{* *}$ & $1,59 * *$ & 93 \\
\hline Colorado $9560^{1}$ & $7042 b$ & 5468 & 8495 & $0,96 n s$ & $-0,34^{*}$ & $0,60^{* *}$ & 83 \\
\hline Braskalb X工 $360^{2}$ & $6990 b$ & 5322 & 8530 & $1,00 \mathrm{~ns}$ & $0,09 \mathrm{~ns}$ & $1,09 \mathrm{~ns}$ & 90 \\
\hline Zeneca $8410^{1}$ & $6930 \mathrm{c}$ & 5306 & 8489 & $1,21 * *$ & $-0,35 * \approx$ & $0,85 \mathrm{~ns}$ & 89 \\
\hline Agromen $2014^{2}$ & 68890 & 5300 & 8355 & $0,89 * *$ & $-0,06 \mathrm{~ns}$ & $0,837 n$ & 85 \\
\hline Colorado $32^{2}$ & $6857 \mathrm{c}$ & 5224 & 8365 & $0,97 n s$ & $0,12 \mathrm{~ns}$ & $0,85 n 8$ & 88 \\
\hline SHS $5050^{2}$ & $6837 \mathrm{c}$ & 5206 & 8343 & $0,92 \mathrm{~ns}$ & $0,15 \mathrm{~ms}$ & $1,08 \mathrm{~ns}$ & 92 \\
\hline Pioneer $30 \mathrm{~F} 80^{\prime}$ & $6835 \mathrm{c}$ & 5076 & 8145 & $1,07 \mathrm{~ns}$ & $-0,35^{* *}$ & $0,7 I^{* *}$ & 82 \\
\hline Colorado $34^{2}$ & $6830 \mathrm{c}$ & 5002 & 8516 & $1,05 \mathrm{~ns}$ & $0,43 * *$ & $1,48 * *$ & 93 \\
\hline Pioneer $30 \mathrm{~F} 88^{1}$ & $6763 \mathrm{c}$ & 5221 & 8186 & $0,94 \mathrm{~ns}$ & $=0,13 \mathrm{~ns}$ & $0,81 \mathrm{~ns}$ & 91 \\
\hline BRS $3101^{2}$ & $6761 \mathrm{c}$ & 4894 & 8472 & $1,07 \mathrm{~ns}$ & $-0,17 \mathrm{~ns}$ & $0,90 \mathrm{~ns}$ & 86 \\
\hline BR $3123^{2}$ & $6743 \mathrm{c}$ & 5057 & 8312 & $0,96 \mathrm{~ns}$ & $-0,15 \mathrm{~ns}$ & $0,80 \mathrm{~ns}$ & 84 \\
\hline$A G 9010^{1}$ & $6659 \mathrm{c}$ & 5280 & 7923 & $0,79 * *$ & $-0,01 n s$ & $0,77 \mathrm{~ns}$ & 85 \\
\hline Colorado $9743^{2}$ & $6573 \mathrm{c}$ & 4981 & 8042 & 0,96 ns & $0,01 \mathrm{~ms}$ & $0,97 \mathrm{~ns}$ & 93 \\
\hline BRS $3060^{2}$ & $6537 \mathrm{c}$ & 4790 & 8149 & $1,01 \mathrm{~ns}$ & *0,2 Ins & $0,80 \mathrm{~ns}$ & 89 \\
\hline $\mathrm{HT}^{2}$ & $6533 \mathrm{c}$ & 4888 & 8052 & $0,94 \mathrm{~ns}$ & $0,2 \mathrm{lns}$ & $1,16 \mathrm{~ns}$ & 86 \\
\hline HT $10^{2}$ & 65060 & 4888 & 8000 & $0,96 \mathrm{~ns}$ & $0,49 * *$ & $1,45 *$ & 94 \\
\hline HT $9^{2}$ & $6503 d$ & 5062 & 7834 & $0,87 * 4$ & $0,11 \mathrm{~ms}$ & $0,98 n 8$ & 31 \\
\hline HT $5^{2}$ & $6183 d$ & 4663 & 7587 & $0,89^{*}$ & $-0,03 n s$ & $0,86 n s$ & 87 \\
\hline $95 \mathrm{HT} 74^{2}$ & $6064 d$ & 4551 & 7459 & $0,90^{*}$ & $0,14 \mathrm{~ms}$ & $1,04 \mathrm{~ns}$ & 92 \\
\hline SHS $4040^{3}$ & 5981d & 4476 & 7368 & $0,92 \mathrm{~ns}$ & $-0,15 \mathrm{~ns}$ & $0,77 \mathrm{~ns}$ & 87 \\
\hline $96{\mathrm{HT} 91^{2}}^{2}$ & $5413 e$ & 3739 & 6958 & $0.95 \mathrm{~ns}$ & $=0,12 \mathrm{~ns}$ & $0,82 \mathrm{~ns}$ & $\$ 0$ \\
\hline Media & 6971 & & & & & & \\
\hline D. M. S. $(5 \%)$ & 711 & & & & & & \\
\hline
\end{tabular}

${ }^{1}$ Híbrido simples, ${ }^{2}$ híbrido triplo e ${ }^{3}$ híbrido duplo. ** $\mathrm{e}$ * Significativamente diferentes da unidade, para $b_{1}$ e $b_{1}+b_{2}$ e zero, para $b_{2}$ a $1 \%$ e $5 \%$ de probabilidade pelo teste $t$ de Student, respectivamente.

Médias seguidas pela mesma letra não diferem entre si pelo teste de Scott Knott a 5\%.
Diversos trabalhos na literatura procuraram enfatizar que o comportamento dos genótipos frente às oscilações ambientais está, em geral, relacionado à sua base genética. Neste contexto, Allard \& Bradshaw (1964) concluíram que populações com base genética mais ampla interagem menos com o ambiente e, conseqüentemente, são mais estáveis. Por outro lado, existem inúmeros trabalhos com a cultura do milho que permitem inferir não haver uma relação fixa quanto à homogeneidade ou heterogeneidade do material e sua estabilidade, pois é possível selecionar materiais mais estáveis em qualquer grupo: variedades, híbridos simples triplos e duplos (Naspolini Filho, 1976; Carvalho et al., 1998 e 2000a). Neste trabalho, confirmou-se mais uma vez esta última hipótese. Naspolini Filho(1976), após fazer uma revisão de alguns trabalhos concernentes à estabilidade de genótipos de milho, comenta que ocorrem diferenças entre os materiais com o mesmo nível de heterozigose e heterogeneidade, quanto à expressão das funções homeostáticas, e que a maior habilidade de auto-regulação dos indivíduos assegurando graus maiores de homeostase é, comprovadamente, uma característica de genótipos específicos, ou seja, nos híbridos essa habilidade advém das linhagens paternais.

Considerando os resultados apresentados, depreende-se que o material ideal preconizado pelo modelo (Cruz et al., 1989), ou seja, aquele que apresenta uma média alta ( $b_{0}$ alto), o $b_{1}$ menor possível (menos exigente nos ambientes desfavoráveis), $\mathrm{e} \mathrm{b}_{1}+\mathrm{b}_{2} \mathrm{o}$ maior possível (responsivos à melhoria ambiental), e variância dos desvios da regressão próxima ou igual a zero (alta estabilidade nos ambientes considerados), não foi encontrado entre os híbridos avaliados. Também não foi encontrado qualquer híbrido que atendesse a todos os requisitos necessários para adaptação nos ambientes desfavoráveis. Nesse caso, o híbrido teria que apresentar uma média alta ( $b_{0}$ alto), o $b_{1}$ e $b_{1}+b_{2}<1$. Mesmo 
assim, percebe-se que os híbridos DK 350, Pioneer 30 F 45 e Cargill 747 podem ser recomendados para essa situação, por apresentarem $\mathrm{b}_{0}$ alto, $\mathrm{b}_{1}<1$, $\mathrm{b}_{1}+\mathrm{b}_{2}$ semelhante à unidade e $\mathrm{R}^{2}>80 \%$. No que tange aos ambientes favoráveis, apenas o híbrido Zeneca 8550 apresentou os requisitos necessários para ser recomendado para essa classe de ambientes ( $b_{0}$ alto, $b_{1}>1, b_{1}+b_{2}>1$ e $\left.R^{2}>80 \%\right)$. Os híbridos Dina 1000, AG 1051, Zeneca 8420, Dina 800 e Dina 440 podem ser recomendados também para os ambientes favoráveis, por apresentarem produtividades altas ( $\mathrm{b}_{0}$ alto), serem exigentes nas condições desfavoráveis $\left(b_{1}>1\right)$, apresentarem estimativas de $b_{1}+b_{2}$ semelhantes à unidade e valores de $\mathrm{R}^{2}>80 \%$. Os híbridos que responderam à melhoria ambiental $\left(b_{1}+b_{2}>1\right)$ e mostraram rendimentos médios altos, $\mathrm{b}_{1}$ semelhantes à unidade e estimativas de $\mathrm{R}^{2}>80 \%$, como o Cargill 333 B e Zeneca 84 E90, são também recomendados para essa classe de ambientes. De especial interesse para a região são também os híbridos de boa adaptação e que apresentam $b_{1}$ tendendo para zero, $b_{1}+b_{2}$ tendendo para a unidade e estimativas de $\mathrm{R}^{2}>80 \%$, a exemplo dos DK 440, AG 9090, Pioneer 30 F 33, AG 8020, Zeneca 8392 e Cargill 909.

\section{Conclusões}

1- A produtividade média alcançada com os híbridos coloca algumas áreas do Nordeste brasileiro em condições de competir, na exploração do milho, com regiões tradicionais do país que utilizam tecnologias modernas de produção, sobressaindo as áreas dos cerrados do Oeste Baiano, o Sul do Maranhão e o Pólo Uruçuí-Gurgéia, no Piauí.

2- O material ideal preconizado pelo modelo não foi encontrado no conjunto avaliado.

\section{Literatura Citada}

ALLARD, R.W.; BRADSHAW, A.D. Implications of genotype environmental interactions in applied plant breeding. Crop Science, Madison, v.4, p.503508, 1964.

ARIAS, E.R.A. Adaptabilidade e estabilidade de cultivares de milho no Estado do Mato Grosso do Sul e avanço genético obtido no período de 1986/87 e 1993/94. 1996. 118f. Tese (Doutorado em Agronomia) - Universidade Federal de Lavras, Lavras.

CARDOSO, M.J.; CAVALHO, H.W.L. de.; LEAL, M. de L. da S.; SANTOS, M.X. dos. Estabilidade de cultivares de milho no Estado do Piauí. Revista Científica Rural, Bagé, v.5, n.1, p.62-67, 2000.

CARDOSO, M.J.; CARVALHO, H.W.L. de.; PACHECO, C.A.P.; SANTOS, M.X. dos.; LEAL, M. de L. da S. Adaptabilidade e estabilidade de cultivares de milho no Estado do Piauí no biênio 1993/94. 3. Revista Científica Rural, Bagé, v.2 n.1 p.35-44, 1997.

CARNEIRO, P.C.S. Novas metodologias de análises de adaptabilidade e estabilidade de comportamento. 1998. 168f. Tese (Doutorado) - Universidade Federal de Viçosa, Viçosa.

CARVALHO, H.W.L. de.; LEAL, M. de L. da S.; SANTOS, M.X. dos.; CARDOSO, M.J.; MONTEIRO, A.A.T.; TABOSA, M.J. Adaptabilidade e estabilidade de cultivares de milho no Nordeste brasileiro. Pesquisa Agropecuária Brasileira, Brasília, v.35, n.6, p.1115-1123, 2000a.

CARVALHO, H.W.L. de.; LEAL, M. de L. da S.; SANTOS, M.X. dos.; MONTEIRO,A.A.T.; CARDOSO, M.J.;CARVALHO, B.C.L. de. Estabilidade de cultivares de milho em três ecossistemas do Nordeste brasileiro. Pesquisa Agropecuária Brasileira, Brasília, v.35 n.9, p.1773-1781, 2000b.

CARVALHO, H.W.L. de.; SANTOS, M.X dos.; LEAL, M. de L. da S.; ALBUQUERQUE, M.M.de.; TABOSA, J.N. Estabilidade de cultivares 
de milho no Nordeste brasileiro no ano de 1996. Revista Científica Rural, Bagé, v.3, n.2, p.2026, 1998.

CRUZ, C.D.; TORRES, R.A. de; VENCOVSKY, $\mathrm{R}$. An alternative approach to the stability .analisys by Silva and Barreto. Revista Brasileira de Genética, Ribeirão Preto, v.12, p.567-580, 1989.

MARIOTTI, I.A.; OYARZABAL, E.S.; OSA, J. M.; BULACIO, A.N.R.; ALMADA, G.H. Analisis de estabilidad y adaptabilidad de genotipos de caña de azucar. I. Interacciones dentro de una localidad experimental. Revista Agronomica del Nordeste Argentino, Tuculman, v.13, n.14, p.105-127; 1976. MONTEIRO A.A.T.; CARVALHO, H.W.L. de.; PACHECO, C.A.P.; SANTOS, M.X. dos.; ANTEROO NETO, J. F.; LEAL, M. de L. da S. Adaptabilidade e estabilidade de cultivares de milho no Estado do Ceará. Revista Científica Rural, Bagé, v.3, n.2, p.1-10, 1998.

NASPOLINI FILHO, W. Variabilidade fenotípica e estabilidade em híbridos simples, híbridos duplos, variedades e compostos de milho. 1976. 68f. Dissertação (Mestrado) - Universidade de São
Paulo, Escola Superior de Agricultura "Luiz de Queiroz", Piracicaba.

RAMALHO, M.A.P.; SANTOS, J.B. dos.; ZIMMERMANN, M.J. de O. Genética quantitativa em plantas autógamas: aplicação no melhoramento do feijoeiro. Goiânia, UFG, 1993, p.131169. (Publicação, 120).

RIBEIRO, P.H.E.; RAMALHO, M.A.P.; FERREIRA, D.F. Adaptabilidade e estabilidade de cultivares de milho avaliadas em diferentes condições ambientais do Estado de Minas Gerais. In: REUNION LATINOAMERICANA DEL MAIZ, 18. 1999, Sete Lagoas. Memórias... Sete Lagoas: EMBRAPA - CNPMS / México: CIMMYT, 1999. p.251-260.

SAS INSTITUTE. SAS/STAT user's guide: version 6. 4. ed. Cary, 1996. v.1.

SCAPIM, C.A.; CARVALHO, C.G.P. de.; CRUZ, C.D. Uma proposta de classificação dos coeficientes de variação para a cultura do milho. Pesquisa Agropecuária Brasileira, Brasília, v.30, n.5, p.683-686, 1995. 